\title{
Article
}

\section{Why athletes say no to doping: A qualitative exploration of the reasons underpinning athletes' decision not to dope}

MacNamara, Á., and Collins, D.

Available at http://clok.uclan.ac.uk/12447/

MacNamara, Á., ORCID: 0000-0002-8110-6784 and Collins, D. ORCID: 00000002-7601-0454 (2014) Why athletes say no to doping: A qualitative exploration of the reasons underpinning athletes' decision not to dope.

Performance Enhancement \& Health, 3 (3-4). pp. 145-152. ISSN 2211-2669

It is advisable to refer to the publisher's version if you intend to cite from the work. http://dx.doi.org/10.1016/j.peh.2015.09.001

For more information about UCLan's research in this area go to http://www.uclan.ac.uk/researchgroups/ and search for <name of research Group>.

For information about Research generally at UCLan please go to http://www.uclan.ac.uk/research/

All outputs in CLoK are protected by Intellectual Property Rights law, including Copyright law. Copyright, IPR and Moral Rights for the works on this site are retained by the individual authors and/or other copyright owners. Terms and conditions for use of this material are defined in the policies page. 
Running head: WHY ATHLETES SAY NO TO DOPING

9

$23 *$ Correspondence concerning this paper should be addressed to Áine MacNamara, 006

24 Greenbank Building, Institute of Coaching and Performance, University of Central

25 Lancashire, PR1 2HE, UK. Email: AMacnamara1@uclan.ac.uk
This is a pre-proof corrected manuscript, as accepted for publication, of an article published by Elsevier in Performance Enhancement \& Health on $28^{\text {th }}$ October 2015, available online:

http://www.sciencedirect.com/science/article/pii/S2211266915000572 Underpinning Athletes' Decision Not To Dope. Áine MacNamara* \& Dave Collins Institute of Coaching and Performance, University of Central Lancashire, UK (1) 7 8 9 0 1 2 
Running head: WHY ATHLETES SAY NO TO DOPING

\section{Abstract}

27 Athletes' motives for choosing not to use Performance Enhancing Drugs (PEDs) are

28 likely to be diverse and complex, including a consideration of biological factors (e.g.,

29 performance advantage), psychological characteristics (e.g., risk taking behavior), and

30 the athlete's social environment (e.g., the opinion and influence of significant others).

31 As such, a multifactorial (bio, psycho, and social) evaluation is important when

32 examining the reasons against usage. The purpose of this study was to examine the

33 reasons athletes cite for not using PEDs. A phenomenological approach was

34 employed and data were collected from athletes $(n=36)$ and coaches $(n=10)$ using

35 semi-structured interviews and analyzed using Interpretative Phenomenological

36 Analysis. Personal and moral standards were identified as key factors that led to

37 decisions to avoid PED. Psychological and social factors (e.g., the role of significant

38 others such as the coach) also play significant roles in decisions to avoid doping.

39 Although anti-doping testing and education is central to anti-doping strategy, athletes'

40 decision not to dope was made independent of, or at least not contingent on these

41 structures. As such, these findings have the potential to inform educational initiatives

42 designed to combat doping in sport outside the usual emphasis on sanctions and

43 testing.

Keywords: biopsychosocial, cheating, anti-doping strategy, decision-making,

45 qualitative 
Running head: WHY ATHLETES SAY NO TO DOPING

\section{Introduction}

Testing and associated sanctions are generally supported as a means of discouraging performance enhancing drug (PED) use in sport. In fact, the risk of getting caught underpins anti-doping policy and its emphasis on the detection and sanctioning of athletes in violation of anti-doping policy. Furthermore, the social impact of "shame" experienced is viewed as another significant deterrent (Bloodworth \& McNamee, 2010). Thus, even though the stance of anti-doping is sometimes questioned on moral grounds of proportionality (i.e., too much emphasis on too few users, less than $2 \%$ of athletes test positive in any given year, WADA, 2009; cf. Kayser et al., 2007), there seems to be a strong and apparently consistent resistance to such usage and support of the systems used to police against it. Despite this, research has consistently shown that the prevalence of doping is much higher than the positive test results show (e.g., Petróczi \& Naughton, 2011; Pitsch \& Emrich, 2012). Furthermore, use of therapeutic user exemptions (TUEs) for asthma and thyroid medications, and the use of similar substances within legal limits for performance enhancing effects has received considerable attention in the media in recent times. Reflecting this, some researchers have suggested that educational strategies focused on prevention and the promotion of abstinence (Mazanov et al., 2011) are needed as opposed, or at least as an addition, to the focus on detection and punishment. This focus on understanding, promoting, and reinforcing the reasons underpinning athletes' decision not to dope seems warranted as both drug testing and sanctioning have been shown to remain static despite reported increases in the usage of PEDs (Petróczi \& Naughton,2011; Pitsch \& Emrich, 2012).

Accordingly, a broader social science understanding of reasons underpinning abstinence from doping would seem sensible in terms of shifting the attention from 
Running head: WHY ATHLETES SAY NO TO DOPING

detection towards an understanding of athletes' decision making process. The decision to dope is a conscious decision but also an emotional, rational, and wellinformed decision. For example, whilst many athletes report satisfaction with their own environment and national situation, they perceive laxity within systems elsewhere in the world as a major problem (Bloodworth \& McNamee, 2010). Indeed, an over-estimation of drug usage may well be a correlational factor with intention to use in some individuals. Attitudes to other, albeit legal, ergogenic aids such as nutritional supplements or even specific, though often medically endorsed, hormonal treatments represents another important facet of the mental model which underpins athlete thinking about usage, those who use, and their own personal intentions (Mazanov et al., 2008). For example, the use of thyroid and testosterone medication for performance enhancing effects is a current hot-topic in elite sport and understanding athletes' decision making process in this regard, together with similarities and differences between this and illegal PED usage, is an under-explored but important area for exploration in understanding doping in sport.

Given the extant picture of the factors which have an influence, a multifactorial (bio, psycho, and social) evaluation is important when examining the reasons against PED usage. Support for this approach comes from evidence for the mediating role of social desirability (Petróczi, 2007) between attitudes toward and susceptibility to engage in PED usage (Gucciardi et al., 2010). From a psychosocial perspective, the "protective" or "encouraging" influences of team dynamics against PEDs have also been demonstrated (cf. Lentillon-Kaestney \& Carstairs, 2010). Furthermore, the effectiveness of testing and sanctions has also been questioned by Strelan and Boeckmann (2006) who suggest that athletes consider their moral beliefs, fear of health impacts and legal consequences when making decisions about PED 
Running head: WHY ATHLETES SAY NO TO DOPING

101 usage. Indeed, there appears to be a theoretical and empirical consensus on critical

102 social-cognitive determinants of doping usage (e.g., Dodge \& Jaccard, 2008; Lucidi et

103 al., 2008).

104 Extending the social dimension, the role of the coach as mediator of the

105 athlete's social environment and the influences therein is an important factor

106 (Huybers \& Mazanov, 2012; Morente-Sanchez \& Zabala, 2013). This research

107 suggests that athletes are more at risk of doping if coaches or senior athletes provide

108 convincing evidence of the immediate benefits. Once again, however, there is a need

109 for further work since reviews clearly show the extra potential insights which such a

110 focus could offer (Backhouse \& McKenna, 2012). Finally, the coach's viewpoint may

111 offer an additional perspective, answering some of the concerns expressed about the

112 limitations of self-report data which, to date, has provided the majority of data on

113 PEDs (Brand et al., 2011). In simple terms, therefore, there is clear evidence for the

114 complex interactions that seem to be associated with uptake of use or even

115 consideration to start, all of which must sensibly be encompassed within any global

116 anti-doping strategy (cf. Stewart \& Smith, 2010).

117 A number of reasons underpinning decisions not to dope have been found in

118 the literature (e.g., Ehrnborg \& Rosén, 2009). These include "doping is cheating and

119 not fair play", the medical risks associated with doping, the perceived impact of

120 doping on performance in particular sports, and the impact which doping has upon the

121 image of a sport (e.g., Mohamed, Bilard \& Hauw, 2013; Erickson, McKenna \&

122 Backhouse, 2014). Theoretical approaches to understanding the psychology of doping

123 have emphasised social-cognitive determinants of use where doping is seen, using the

124 theory of planned behaviour (Ajzen, 1991), as a volitional behaviour depending on

125 the athlete's intentions to use PEDs, which are influenced by attitudes, expected 
Running head: WHY ATHLETES SAY NO TO DOPING

126 social approval and perceived behavioural control. Furthermore, and as discussed

127 previously, the importance of individual views about the approval of significant

128 others, PED use amongst peers (Wiefferink et al., 2008) as well as the individual's

129 confidence about resisting social pressure (Lucidi et al., 2008; Erickson et al., 2014)

130 have all been shown to play a role in understanding PED usage. Despite this

131 understanding, however, the testing of these ideas amongst elite athletes has been

132 scarce and the predominant emphasis has been on reasons why athletes do dope (e.g.,

133 Kirby et al., 2011) rather than on the reasons that they don't. Dodge and Jaccard

134 (2008) present an important advance on these ideas and suggest that abstinence is a

135 "viable, independent, behavioural alternative in some decision making contexts" (p.

136 710). Using a sample of adolescent athletes, this research found that the reasons

137 underpinning decisions not to dope were not merely the inverse of the reasons cited

138 for doping and that focusing on emotive and affective beliefs shown to influence

139 intention not to dope within intervention programs may affect the use of PEDs

140 (Dodge \& Jaccard, 2008).

The emerging picture may lack clarity, however. An obvious limitation of

142 many studies to date is that data is often not based on truly elite samples, with various

143 studies conducted with high school (e.g., Laure et al., 2004), adolescent (e.g., Laure \&

144 Binsinger, 2007), or collegiate (e.g., Petroczi, 2007) athletes. Consequently, further

145 work is indicated to confirm these findings with elite populations. As such, it would

146 be valuable to see if the decision to not use PEDs is impacted or moderated by the

147 elite status of the athlete and their perception of the environment in which they

148 perform. If so, and based on data with genuine elites (e.g., Moran et al., 2008), there

149 are strong indications that programs utilizing accurate and empirically justified

150 information could prove a strong feature of a deterrent program. 
Running head: WHY ATHLETES SAY NO TO DOPING

151

152

153

154

155

156

157 Reflecting these issues, the purpose of this study was to examine the reasons athletes cite for not using PEDs. Previous research has shown attitudes towards doping vary by sex, with males at a higher risk than females and sport, risk of doping is highest in speed and power sports (both factors highlighted by Alaranta et al., 2006). Further, Vangrunderbeek (2011) reports a shift in attitude over time from 'zero tolerance' to a more lenient attitude towards doping in sport as athletes age. Reflecting this, we were also interested in exploring whether the reasons not to use PEDs might vary against a number of key factors including age, sport, and level of performance. Given the important impacts demonstrated for psychosocial milieu, this study was delimited to an examination of athletes from a British and Irish culture. As the aim of this study was to explore athletes' personal experiences of decision-making about PEDs, a phenomenological approach was employed.

\section{Methods}

\section{Design}

Data were collected using semi-structured interviews and analyzed using Interpretative Phenomenological Analysis (IPA; Smith, 1996), as this approach allows rigorous exploration of idiographic subjective experiences and social cognitions. Essentially, IPA explores how people ascribe meaning to their experiences in their interactions with the environment (Smith et al., 1999)

\section{Participants}

A purposive sample of athletes $(n=36)$ and coaches $(n=10)$ were recruited from a range of sports (i.e., power, endurance and team sports) and backgrounds. Athletes were all high-level participants in their chosen sport (defined as participation at a world-level (e.g., World Championship or Olympic Games for the power and endurance sports; International for team sports) and declared that they had not taken 
176 PED during their sport careers (see Table 1). This purposeful sample was an

177 important consideration in order to examine the elite viewpoint. A range of sports was

178 purposefully sampled in order to identify the extent to which findings, and

179 consequently policy and strategy, could be generalizable and impactful. The coaches

180 had, at least, 15 years' experience coaching at a world-class level (e.g., (e.g., World

181 Championship or Olympic Games for the power and endurance sports; International

182 level for team sports)

183 Procedure

Following research ethics board approval, coaches and athletes from a range

185 of sports who met the sampling criteria were recruited through personal contact, either

186 directly or through gatekeepers. The study was explained to participants, and consent

187 forms were distributed to those who expressed interest. A semi structured interview

188 approach similar to the majority of IPA studies was adopted (Smith \& Osborn, 2003).

189 The interview schedule was not intended to be prescriptive and instead, the interview

190 guide was used as a prompt and a basis for conversation. Consistent with the IPA

191 approach, participants were considered to be the experts and it is the meaning that

192 they attribute to their experiences that was of interest (Smith, 1996). As such,

193 participants were allowed to take the lead during the conversation and direct the flow

194 of the interview. The interviewer was an experienced sport psychologist who has over

19530 years' experience working at the highest level of sport in a variety of roles. This

196 experience and understanding of elite sport, coupled with a clear separation from the

197 respective sports organizations, training groups, and anti-doping agency, were

198 important factors in developing rapport with the participants and ensuring that they

199 were comfortable responding to questions. All the interviews were recorded and

200 transcribed verbatim to produce an accurate record of the interviews. Excluding 
Running head: WHY ATHLETES SAY NO TO DOPING

201 introductions, explanations and initial conversation to build rapport, the interviews all 202 lasted between 35 and 55 minutes.

\section{Data Analysis}

Data were independently analyzed using Smith and Osborn's

205 recommendations for IPA analysis (2003). First, all transcripts were read and reread

206 so that the researchers could become familiar with each participant's account. At this

207 stage, initial notes of thoughts, observations, and reflections were recorded in the

208 right-hand margin of the interview transcript and shared with the research team. In a

209 second reading, the left-hand margin was used to identify themes that captured the

210 essential qualities of the interview and connections were made between the emergent

211 themes and researcher interpretations (Smith \& Osborn, 2003). As a result, a list of

212 subordinate themes and codes were complied, with the aim of providing an overall

213 structure to the analysis by relating the identified themes into clusters and to identify

214 super-ordinate categories that suggest a hierarchical relationship between them.

215 Throughout this process, checks were made with the original transcript and the

216 interviewer's field notes to ensure that connections still worked with the original data

217 and that the analytic accounts could be traced back to recognizable core accounts. In

218 cases where this step identified a disagreement, each investigator reread the original

219 transcript, discussed the coding, and a consensus was reached. Disagreement was

220 evident in less than $15 \%$ of codes and all issues were resolved following discussion.

221 Once the analysis was completed for one transcript, a second transcript was coded.

222 The table of themes was used to code similar meanings in the same categories, and

223 was expanded to incorporate new ideas as they emerged. During this phase, emergent

224 themes were continually compared back to the original transcripts to ensure

225 consistency. Once this process had been completed for all the transcripts, the research 
Running head: WHY ATHLETES SAY NO TO DOPING

226 team reread the transcripts to ensure that all themes were coded consistently (Smith \&

227 Osborn, 2003). As expected with this form of analysis, some of the emergent themes

228 reflected the content of the interview schedule, while others emerged from the

229 participants' novel responses. The super-ordinate themes and their sub-ordinate

230 components are presented in Table 2 along with a short verbatim account that

231 illustrates each super-ordinate theme.

232 Ensuring Trustworthiness and Credibility

233 A number of steps were taken to enhance the study's trustworthiness (Lincoln

$234 \&$ Guba, 1985). Bracketing, which involved the researchers keeping a reflective diary

235 to help bracket their personal experiences and consider the influence of personal

236 values, was used (Nicholls et al., 2005). Furthermore, and also ensuring that the

237 authors remained cognizant of their assumptions and presumptions, an independent

238 "critical friend" was used throughout the data analysis process by supporting in-depth

239 critique and investigation of the emerging interpretation, discoveries and explanations

240 (Faulkner \& Sparkes, 1999). Credibility was also enhanced in a number of ways

241 including the sample size employed, having two investigators involved in each level

242 of analysis, and having researchers with significant experience in performance sport

243 involved in the study (Sparkes, 1998).

\section{Results}

Table 2 highlights the range of factors underpinning athletes' decision making

246 about PEDs. All participants mentioned each of the super-ordinate themes during

247 their interviews. Sub-ordinate themes were only included when data from at least $75 \%$

248 of the participants could be attributed to the theme. As such, the findings reported

249 represent consensus amongst the group.

250 Anti-Doping Testing and Associated Sanctions 
Running head: WHY ATHLETES SAY NO TO DOPING

Despite the emphasis placed by WADA and National Governing Bodies of

252 Sport on anti-doping testing and associated sanctions, these factors were not reported

253 as central to athletes' decision to avoid doping. Interestingly, although athletes were

254 cognizant of the testing procedures in place, many suggested that there were "ways

255 around the testing procedures... if you want to do it, there are ways to dope without

256 getting caught" (Endurance sport athlete, International, male). Furthermore, the

257 majority of participants suggested that they still would not take PEDs even if the anti-

258 doping testing procedures were removed. Illustrating this, one premiership rugby

259 player described how “it wouldn't make any difference to me...I could go away to

260 visit a mate in South Africa for six weeks in the summer and come back a lean

261 sprinting machine, seven kilos up in weight and I know I wouldn't get caught for it.

262 But I still wouldn't do it”.

There did appear to be some differences across the different sports, perhaps

264 reflective of the level of anti-doping testing carried out. Track and field athletes

265 suggested that they would likely be tested and that this acted as somewhat of a

266 deterrent - 'I've been tested in the past, and you still cack yourself because even

267 though I know I am clean, you think what if something shows up, what if I took

268 something without knowing...so it does keep you on your toes in that respect'.

269 (International Athlete). However, many of these athletes suggested that there were

270 many in their sport who were 'way ahead of the testers...I mean, they know how to get

271 away with it' - '...you read about people and you hear it as well, that certain things

272 can be out of your system before they test, or they can't test for certain things yet, so

273 people are getting away with it' (Endurance athlete, International level).

274 The team sport athletes, rugby players and footballers for example, suggested

275 that testing was not a deterrent since testing was not that prevalent in their sport ' ...it 
Running head: WHY ATHLETES SAY NO TO DOPING

isn't the testing that stops me, we rarely get tested, so yeah, it is not that I don't take drugs because I might get caught ... that isn't the reason' (Rugby player, International).

Anti-Doping Education. Participants also suggested that anti-doping education was not an influencing factor in their decision not to take PEDs. In most cases, participants reported that they had made their decision about doping long in advance of their first attendance at a workshop and described how these educational sessions "just educated you on the testing procedures ...they don't really get you to think about the reasons why you should or shouldn't" (Judo player, International). Although most participants reported that anti-doping education was useful in that it informed them about policies and procedures - "I think the information was good in that way...it gave me a clear understanding of what to watch out for when you are taking stuff...the Sudafed and all that..." (Track and field athlete, International) it didn't impact on their decision-making process about taking illegal PEDs - "I don't think it was that effective really...I formed an opinion long before any of these workshops and I would stick to these" (Judo player, International). These results suggest that the traditional emphasis on education, testing, and sanctions in anti-doping campaigns does not appear to be a significant influencing factor on these individual's decisions about PEDs.

\section{Personal Ethical Standards}

In contrast to the comparatively weak role played by education and testing, the key factor that influenced decision-making about PEDs centered on the athlete's moral stance about doping in sport. Participants strongly suggested that doping was a moral decision, typified by this athlete's explanation that, irrespective of whether the athlete would get caught, it is wrong and "cheating". Typifying this, one development 
Running head: WHY ATHLETES SAY NO TO DOPING

301 level endurance athlete stated that "I have friends who don't even get tested, who could easily take drugs, get themselves to a reasonable performance level and stop

303 because they will never get caught. But they don't for the same reason that I don't,

304 because they feel like they are cheating themselves". Interestingly, the participants

305 described this as "a line that I wasn't prepared to cross" with one international level

306 Judo player suggesting that she "doesn't want to cheat myself, and I don't want to

307 cheat the other four fifths of people that are competing with me, the ones that are

308 competing without doping, I don't want to cheat myself and I don't want to cheat

309 them".

310 Participants were also asked to compare and contrast doping with other

311 "cheating" behaviors in their sport. Of course, cheating is difficult to define in this

312 context but can be understood as violating the explicit or implicit nature of the rules

313 of the competition in order to gain an advantage (Lee et al., 2007); simply,

314 professional fouls or gamesmanship. Interestingly, participants suggested that doping

315 was a significantly worse offense than other forms of cheating such as diving in

316 football, punching in rugby, or psyching out your opposition in athletics

317 "Punching, getting someone at the bottom of a ruck, all those things are

318 cheating, like to the letter of the law. But not one rugby player plays the game

319 to the letter of the law, you are always looking for the little advantage. So you

320 are constantly pushing that line but I think that that is different to taking

321 drugs, that is what you do in the heat of battle, I think there is a line in sport

322 and I know that I wouldn't cross it'. (International rugby player)

323 Although the athletes acknowledged that these behaviors were outside the rules of the

324 sport, they suggested that they were part of the game whereas doping was outside the

325 spirit of the sport and not acceptable. 
Running head: WHY ATHLETES SAY NO TO DOPING

This moral complexity was an interesting basis for athletes' decision about "cheating" behaviors in their sport. Although they stated that their decision about PEDs was morally based, the decision making underpinning other aspects of the participants' behavior in the sport had a more rational underpinning. The key message that emerged from participants in this regard was that there was a personally enforced ethical line that they wouldn't cross to gain an "unfair advantage” against their peers. There also appeared to be significant age effects apparent in athletes' attitudes towards, though not necessarily their usage of, PEDs. A minority of older athletes and coaches (then as athletes) admitted to taking PEDs during their early career and recognized the temptation of this. Conversely, the younger cohort of athletes strongly articulated their stance and stated how they would not take PEDs due to their personal ethical standards. As such, and perhaps somewhat surprisingly given the increasing competiveness of elite sport, the younger athletes displayed a much stronger antidoping stance, grounded by their personal morals and ethics, than the older athletes

340 and coaches. However, there was significant complexity evident underpinning

341 athletes' decision making about performance enhancing substances, both legal and 342 illegal, and these will be explored further in the next section.

\section{$343 \quad$ Illegality of Substances}

The central role that morals seemed to play in the athletes' decision making

345 was interesting and went beyond the use of PEDs. The legality of substances was an

346 important factor in the athletes' decision making with all the participants suggesting

347 that legal nutritional aids are not cheating "because WADA says so!” However,

348 although all the participants spoke about the legality of substances as an important

349 factor in their decision, this was actually a complex issue. For example, when athletes

350 were probed about whether they would take medical supplements to achieve above 
Running head: WHY ATHLETES SAY NO TO DOPING

351 normal, though still legal, levels (e.g., thyroid manipulation) the majority suggested

352 that they wouldn't be comfortable, describing this type of supplementation as also

353 "unethical" and "cheating". For example, one international level endurance athlete

354 when asked about whether he would take testosterone to boost his levels responded: "I don't know, I guess if the doctor said I needed to, if it was healthy. If I went to a normal GP and they suggested that I took it, not anything to do with the sport, then I would take it. But if I went to a doctor from [name of NGB] and they said, take it, it will boost your performance, then I would be like well, why do you want me to do that...I would feel different about it if it was only performance enhancing...”

361 In fact, this idea of equality was another reason athletes cited for not taking PEDs, 362 describing how other, legal, substances were acceptable because "I feel that everyone 363 has access to that sort of dietary stuff" and "if it is allowed and everyone is doing it 364 then I think it's alright. If everybody is on the same playing field then its fine but if 365 people are taking stuff that does a bit more than help you recover then I think there is 366 a big difference" (Track and field athlete, development level). As described in the previous section, age effects were apparent in athletes' and 368 coaches' responses to these questions. For example, when a younger international 369 level endurance athlete was asked "would you take supplementary testosterone to get 370 your levels up to a normal, legal...would that be cheating?' he replied, "No, that is 371 not acceptable, if it is specifically targeted to get you to the limit, the legal limit, then 372 I would say that is cheating, I wouldn't do it'. However, when responding to a similar 373 question, an older coach suggested that "there is stuff that sails a little close to the 374 wind, thyroid manipulation and things, it is legal but still kind of iffy...if it would help 375 an athlete and it was legal, maybe even if I had reservations, I would want the athlete 
Running head: WHY ATHLETES SAY NO TO DOPING

376

377

378

379

380

381

382

383

384

385

386

387

388

389

390

391

392

393

394

to have it" (Track and field coach). This potentially related age and role (i.e., coach or athlete) effect deserves further clarification but should have important implications for the design and delivery of anti-doping policy and education.

\section{The Role of Significant Others}

A number of key psycho-social influences emerged as playing a central role in athletes' decision making about PEDs. Firstly, the importance of the training group and culture of their sport was cited as fundamental to athletes' decision not to take PEDs. The participants described how doping was "culturally inevitable" in other countries and sport systems but was not part of their involvement in sport. One developmental level judo player suggested that "it [doping] is not part of what I understand as traditional Judo culture. We are quite traditional in this group, we have a traditional background, a lot of what we take as our culture is from [name of coach] and before him and because of that, no I would never consider doping". As such, anticipated feelings of shame and guilt associated with doping were cited as key reasons underpinning the decision not to dope with a number of participants suggesting that they would be letting significant others who helped them achieve in their sport down. For example, one international endurance athlete described how he "came from a very strong family background, and to my family through that if I got busted for a positive test...I could never, I could never even consider that”.

\section{Psycho-social Environment}

The protective mechanism of the athletes' training environment certainly appeared to influence their decision, with significant others, including parents, coaches and peers, all playing a role in the athletes' decision-making. Interestingly, many of the participants emphasized the role of parents in guiding their decisions about PEDs and how their upbringing instilled those values from an early age. 
Running head: WHY ATHLETES SAY NO TO DOPING

401 Typifying this, one international level footballer described how "yeah that comes from my family, you shouldn't win by cheating and I think that is what I have been

403 taught and that is how I like to win". Reflecting the role played by significant others,

404 many of the participants suggested that they trusted the actions of coaches and other

405 medical and sport science support staff in guiding their decision about substances. For

406 example, another international level footballer commented that "you put your trust in

407 a lot of the people around you, and you hope that they give you the right advice".

408 However, despite the importance placed on significant others, and the rules governing

409 what is legal or not, participants all stressed that it was their individual decision to

410 take or refuse PEDs. Supporting this, one international level rugby player described

411 how "this is my line, someone else's line might be different, but this is my line and I

412 won't cross it". Nonetheless, the importance of reference group opinion, peers and

413 significant others' approval or disapproval of doping, does appear to play an

414 important role in athletes' decision-making about doping.

\section{Discussion}

Testing and anti-doping education is central to anti-doping strategy (WADA,

417 2009). However, the results of this qualitative study suggest that athletes' decision not

418 to dope was made independent of, or at least not contingent on, these structures. This

419 reflects other evidence which suggests that anti-doping testing and sanctions do not

420 play a significant role in athletes' decision not to dope. Instead, the individual's

421 personal and moral standards, and the influence of their psycho-social environment

422 appear to be the key factors underpinning their decision about doping (Erickson et al.,

423 2014; Petrozci, 2007; Wiefferink et al., 2006). However, this moral reasoning

424 appeared to be more complex than "it is just against the rules so I won't do it". The

425 athletes suggested that they had their own "moral compass" that guided their 
Running head: WHY ATHLETES SAY NO TO DOPING

426 decisions about both PEDs and other legal performance enhancing substances (Dodge

427 \& Hoagland, 2011; Strelan \& Boeckmann, 2006). This was illustrated by the athletes'

428 suggestion that they would not take legal substances just to gain a performance

429 enhancing effect even if these were allowed. Further, the participants described the

430 shame that would be associated with getting caught doping and this was very much

431 described in terms of a moral emotion and a failure to live up to the norms and

432 expectations of their social group (Eisenberg, 2000). The ability to influence athletes'

433 moral compass would seem an effective way to influence decision-making about

434 PEDs in sport. Interestingly, the participants were very strong in their stance that they

435 would prefer to compete, and perhaps not win, as a "clean" athlete than be more

436 successful by taking PEDs (Laure et al., 2004).

When athletes' attitudes to doping, compared to other forms of cheating in

438 their sport, are examined a number of interesting issues emerge. Although the

439 participants suggested that they would engage in some forms of cheating when it was

440 within the spirit of the sport (e.g., attempts to 'psych' opponents out or illegal

441 tackling) the degree of rationality in terms of decision making about PEDs was

442 interesting (Backhouse et al., 2007) - even if the athletes weren't going to get caught

443 and they were assured their performance would improve, they still reported that they

444 wouldn't take PEDs. Again, this points to the importance of attitudes and morals as a

445 key feature of the decision-making process (Haugen, 2004).

446 The differences across different age cohorts is another important issue that

447 emerged from the results and is consistent with previous research (e.g., Mazanov et

448 al., 2008). For example, there appeared to be a significant difference in older and

449 younger participants' responses to the questions about illegality of substances with the

450 younger cohort strongly suggesting that even if certain substances were legal (or not 
Running head: WHY ATHLETES SAY NO TO DOPING

451 tested for) they would not take them as this crossed their "personal moral compass".

452 Conversely, the older cohort was not as strong in their conviction about this and

453 suggested that "as long as it was legal, it was ok". Given the rapid development of

454 PEDs and the difficulty of maintaining an efficient testing program that can

455 adequately test of all PEDs the role of personal ethical and moral standards in

456 younger athletes should be an important avenue for exploration for anti-doping

457 agencies.

Unlike some evidence from the literature (e.g., Goldman \& Klatz, 1992),

459 athletes did not report health risks as a significant factor in their decision not to dope.

460 In fact, the negative health risks (both short and long term) were not seen as

461 influencing factors with most athletes suggesting "I haven't even thought about it, the

462 health implications wouldn't have crossed my mind". Although the lack of attention

463 to long-term health risks associated with PEDs may be expected within a young

464 population, such as that sampled for this study (Ehrnborg \& Rosén, 2009), short-term

465 health implications were also not seen as a significant factor in the athletes' decision-

466 making. As such, the significant factors influencing the athletes' decision not to dope

467 appear to be their personal moral and ethical standards rather than a "cost versus

468 benefit" evaluation of doping. Personal moral beliefs therefore seem to act as a

469 preventing factor for doping (Strelan \& Boeckmann, 2006).

470 Interestingly, the participants were realistic that, at least in some sports, many

471 competitors were taking PEDs and that success at the world level was difficult for

472 "clean" athletes. Despite this, the overwhelming majority reported that they wouldn't

473 take PEDs, not primarily because they were banned or the likelihood of getting

474 caught, but because cheating in this manner was against their personal ethical

475 standards. This is not to say that the athletes wouldn't cheat in other ways (e.g., 
Running head: WHY ATHLETES SAY NO TO DOPING

476 diving, shirt pulling), defined by the athletes as "within the spirit, if not the rules of

477 the game”. In fact, the athletes' stated reluctance to take legal supplements for purely

478 performance enhancing reasons is interesting against the growing trend worldwide for

479 such supplementation. The athletes suggested that this crossed a line of fairness but

480 did recognize that there "shades of grey" in terms of this debate. For example, the

481 participants recognized that other legal supplements such as creatine or caffeine also

482 have performance enhancing effects but suggested that they were comfortable with

483 these because they are available to all athletes. However, the complexity underpinning

484 this decision making is worthy of attention as it, no doubt, has a significant impact on

485 the athletes' attitudes to different performance enhancing supplements. In fact, the

486 complexity of this issue is evident in the "hypocritical" stance taken by some athletes

487 about one substance and another suggesting that athletes' attitudes to PEDs is not as

488 clear cut as whether a substance is legal or not.

489 The athletes' psychosocial environment, and the role of significant others, was

490 also shown as a key factor underpinning their decision about PEDs. As found

491 elsewhere in the anti-doping literature (e.g., Bird \& Wagner, 1997), the external

492 pressures of social and moral expectations acted as a deterrent with coaches, the

493 norms of the training group, and peers especially important in this influence. As such,

494 interventions and anti-doping strategies that work at group levels would seem an

495 efficacious way to influence decision making about taking PEDs. In fact, the

496 traditional anti-doping education procedures were described by the participants as

497 "not particularly useful" outside the focus on procedures and systems. Instead,

498 influencing the subculture of a sport or training environment may be more effective.

499 This was particularly evident in the current results with athletes describing how the

500 anti-doping ethos of their training group, sport, and country played a role in their 
Running head: WHY ATHLETES SAY NO TO DOPING

501 decision (Mazanov \& Huybers, 2010; Strelan \& Boekmann, 2003). The sport's

502 culture has been shown to be influential in precipitating PED use (Kirby et al., 2011)

503 as described by admitted dopers. Individuals strive to show solidarity with peers and

504 enhance their group identity by conforming to group norms. Therefore, altering

505 expectations and group norms about doping would seem a salient way to impact PED

506 usage. This might be especially important from a developmental perspective given

507 that many factors such as role models, vulnerability to peer pressure, and attitudes

508 change as athletes move from one developmental stage to another (Petróczi \&

509 Aidman, 2008).

510 As found elsewhere in the literature, participants suggested that doping was

511 not a widespread problem within their training group or country and that there was an

512 "anti-doping culture" in UK / Irish sport. However, there were repeated references to

513 the extent of the problem in other countries. In fact, the track and field and endurance

514 athletes as well as the rugby players suggested that there was systematic and

515 organized doping in other countries, similar to the "sporting xenophobia" described

516 by Bloodworth and McNamee (2010). Although this "doping dilemma" has been

517 suggested to be a driving factor in PED usage, since the associated suspicion that

518 everyone else is using PEDs drives athletes to use to compete under the same

519 circumstances, this was not the case in this study. Instead, the participants' personal

520 moral standards, reinforced by their psycho-social environment, were the driving

521 factor in their decision not to dope. This finding has interesting implications for anti-

522 doping policies. Given the protective influence that coaches, significant others and the

523 social milieu appear to play in an athletes' decision not to dope, emphasis at this

524 social level would seem important. 
Running head: WHY ATHLETES SAY NO TO DOPING

Of course, there are a number of limitations to this study that must be

highlighted and considered. Firstly, this study is based on participants' self-reported accounts and, given the nature of the topic, the findings must be interpreted in light of this and the possibility that participants were not honest in their responses, despite the steps taken during the data collection process to overcome this limitation. We also acknowledge that the findings of this study are delimited to an Irish and British population. Given that the social environment, and by extension cultural milieu, has been shown to play a significant role in athletes' decision making, it would be worth exploring the extent that these findings are generalizable to other countries, cultural contexts, and indeed other sports (e.g., aesthetic sports for example). Finally, we did not explore differences between male and female athletes in this study due to the relatively small number of females recruited to participate (cf. Alaranta et al., 2006). However, given that males tend to have a more permissive attitude towards doping (Bloodworth et al., 2012), as well as the paucity of research on females' experiences of doping at elite levels of sport, it would be interesting from both an academic and applied perspective to further examine the reasons females "say no" to doping as these may potentially differ from their male counterparts.

The findings from this study suggest that there are interesting implications for emphasizing the importance of abstinence, "saying no", within anti-doping policy (cf. Dodge \& Jaccard, 2008). These results support the literature suggesting that there are different behavioral outcomes associated with abstinence from risky behavior compared to engaging in risky behavior and these are manifested in an individual's attitudes, beliefs and social norms (Dodge \& Jaccard, 2008). Importantly, many of the reasons underpinning abstinence from PED usage were affective, emotional and social and targeting these in doping prevention strategies should be an important 
Running head: WHY ATHLETES SAY NO TO DOPING

550 consideration. Reflecting this, anti-doping strategies should benefit from campaigns

551 that emphasis the positive effects of abstinence rather than the negative effects of

552 engaging in doping or stressing the prevalence of PED usage.

553 
Running head: WHY ATHLETES SAY NO TO DOPING

\section{References}

Ajzen I. The theory of planned behavior. Org Behav Human Dec Proc, 1991; 50: 179-211

Alaranta A. Alaranta H. Holmila J. Palmu P. Pietila K. I. Helenius. Self reported attitudes of elite athletes towards doping in sport: Differences between type of sport. Int J Sports Med 2006; 27: 842-846.

Backhouse SH. Ali A. Biddle SJH. Williams C. Carbohydrate ingestion during prolonged high-intensity intermittent exercise: Impact on affect and perceived exertion. Scand J Med Sci Spor 2007; 17: 605-610.

Backhouse SH. McKenna J. Reviewing coaches' knowledge, attitudes and beliefs regarding doping in sport. Int J Spor Sci Coaching 2012; 7:167-175.

Bird EJ. Wagner GG. Sport as a common property resource: A solution to the dilemma of doping. J Conflict Resolut 1997; 41: 749-766.

Bloodworth AJ. Petróczi A. Bailey R. Pearce, G. McNamee MJ. Doping and supplementation: the attitudes of talented young athletes. Scand J Med Sci Sport 2012; 22(2): 293-301.

Bloodworth A. McNamee M. Clean Olympians? Doping and anti-doping: The views of talented young British athletes. Int J Drug Policy 2010; 21(4): 276-282.

Brand R. Melzer M. Hagemann N. Towards an implicit association test (IAT) for measuring doping attitudes in sports: Data-based recommendations developed from two recently published tests. Psychol Sport Exerc 2011; 12: 250-256.

Dodge T. Jaccard JJ. Is abstinence an alternative? Predicting adolescent athletes' intentions to use performance enhancing substances. J Health Psychol 2008; 13: 703-711. 
Running head: WHY ATHLETES SAY NO TO DOPING

578

579

580

581

582

583

584

585

586

587

588

589

590

591

592

593

594

595

596

597

598

599

600

601

602

Dodge T. Hoagland MF. The use of anabolic androgenic steroids and polypharmacy: A review of the literature. Drug Alcohol Depen 2011; 114: 100-109.

Ehrnborg C. Rosén T. The psychology behind doping in sport. Growth Hormone and IGF Research 2009; 19(4): 285-292.

Eisenberg N. Emotion, regulation and moral development. Annu Rev Psychol 2000; 51: 665-697.

Erickson K. McKenna J, Backhouse SH. A qualitative analysis of the factors that protect athletes against doping in sport. Psych Sport Exer 2014; 16: 149 - 155.

Faulkner G. Sparkes A. Exercise as therapy for schizophrenia: An ethnographic study. J Sport Exer Psych 1999; 21: 52-69.

Goldman R. Klatz R. Death in the Locker Room: Drugs \& Sports. $2^{\text {nd }}$ ed. Chicago, Ill.: Elite Sports Medicine Publications; 1992.

Gucciardi DF. Jalleh G. Donovan RJ. Does social desirability influence the relationship between doping attitudes and doping susceptibility in athletes? Psychol Sport Exerc 2010; 11: 479-486.

Haugen KK. The performance-enhancing drug game. J Sport Econ 2004; 5: 67-86.

Huybers T. Mazanov J. What would Kim do: A choice study of projected athlete doping considerations. J Sports Man 2012; 26: 322-331.

Kayser B, Mauron A. Miah A. Current anti-doping policy: A critical appraisal. BMC Med Ethics 2007; 28: 8-2.

Kirby K. Moran A. Guerin S. A qualitative analysis of the experiences of elite athletes who have admitted to doping for performance enhancement. Int J Sport Pol Polit 2011; 3: 205-224.

Laure P. Binsinger C. Doping prevalence among preadolescent athletes: a 4-year follow-up. Br J Sports Med 2007; 41: 660-663. 
Running head: WHY ATHLETES SAY NO TO DOPING

603

604

605

606

607

608

609

610

611

612

613

614

615

616

617

618

619

620

621
Laure P. Lecerf, T. Friser A. Binsinger C. Drugs, recreational drug use and attitudes towards doping of high school athletes. Int J Sports Med 2004; 25: 133-138

Lee MJ. Whitehead J. Ntoumanis N. Development of the attitudes to moral decisionmaking in youth sport questionnaire (AMDYSQ). Psychol Sport Exerc 2007; 8: 369-302.

Lentillon-Kaestner V. Carstairs C. Doping use among young elite cyclists: A qualitative psychosociological approach. Scand J Med Sci Spor 2010; 20(2): 336-345.

Lincoln YS. Guba EG. Naturalistic Enquiry. Newbury Park, CA: Sage Publications; 1985.

Lucidi F. Zelli A. Mallia L. Grano C. Russo P. C. Violani. The social-cognitive mechanisms regulating adolescents' use of doping substances. J Sports Sci 2008; 26: 447-456.

Mazanov J. Huybers T. An empirical model of athlete decisions to use performance enhancing drugs: qualitative evidence. Qual Res Sport Exer 2010; 2(3): 385402.

Mazanov J. Huybers T. Connor, J. Qualitative evidence of a primary intervention point for elite athlete doping. J Sci Med Sport 2011; 14: 106-110.

Mazanov J. Petroczi A. Holloway A. Bingham J. Predicting supplement use among high performance UK athletes. J Sci Med Sport 2008; 11: 185-190.

Mohamed S. Bilard J. Hauw D. Qualitative and hierarchical analysis of protective factors against illicit use of doping substances in athletes calling a national anti-doping phone-help service. Montenegrin J Sport Sci Med 2013; 2(2):2125 . 
Running head: WHY ATHLETES SAY NO TO DOPING

Moran A. Guerin S. Kirby K. MacIntyre, T. The development and validation of a doping attitudes and behaviour scale. Lausanne, Switzerland: World AntiDoping Agency; 2008.

Morente-Sanchez J. Zabala M. Doping in sport: A review of elite athletes' attitudes, beliefs and knowledge. Sports Med 2013; 43: 395-411.

Nicholls AR. Holt NL. Polman RCJ. A phenomenological analysis of coping effectiveness in golf. Sport Psychol 2005; 19:111-130.

Petróczi A. Attitudes and doping: a structural equation analysis of the relationship between athletes' attitudes, sport orientation and doping behavior. Subst Abuse Treat Prev Pol 2007; 2: 34-44.

Petróczi A. Aidman E. Psychological drivers in doping: The life-cycle model of performance enhancement. Subst Abuse Treat Prev Policy 2008; 3:7.

Petróczi A. Naughton DP. Impact of multidisciplinary research on advancing antidoping efforts. Int J Sport Pol Polit 2011; 3: 235-259.

Pitsch W. Emrich E.The frequency of doping in elite sport: Results of a replication study. Int Rev Soc Sport 2012; 47: 559-580.

Smith JA. Beyond the divide between cognition and discourse: Using interpretive phenomenological analysis in health psychology. Psychol Health 1996; 11 : 261-271.

Smith JA. Jarman M. Osborn M. Doing interpretive phenomenological analysis. London: Sage Murray \& Chamberlain; 1999. Chapter 14, Qualitative Health Psychology: Theories and Methods; p. 218-240.

Smith JA. Osborn M. Interpretive phenomenological analysis. London: Sage Smith; 2003. Chapter 4, Qualitative Psychology: A Practical Guide to Research methods; p. 53-81. 
Running head: WHY ATHLETES SAY NO TO DOPING

652

653

654

655

656

657

658

659

660

661

662

663

664

665

666

667

668

669

670

Sparkes A. Validity in qualitative inquiry and problems of criteria: Implications for sport psychology. Sport Psychol 1998; 12: 363-386.

Stewart B. Smith ACT. The role of ideology in shaping drug use regulation in Australian sport. International Rev. For The Sociology of Sport 2010; 45(2): 187-198.

Strelan P. Boeckmann RJ. Why drug testing in elite sport does not work: perceptual deterrence theory and the role of personal moral beliefs. J Appl Soc Psychol 2006; 36: 2909-2934.

Vangrunderbeek H. Student attitudes towards doping in sport: Shifting from repression to tolerance. Int Rev Soc Sport 2011; 3: 346-357.

Wiefferink C.H. Detmar S.B. CoumansT. Vogels, B. T.G.W. Paulussen. Social psychological determinants of the use of performance-enhancing drugs by gym users. Health Edu Res 2008; 23:70-80

Wiefferink K. Detmar S. de Hon O. Vogels T. Paulussen T. Proceedings of the International Conference on Ethics and Social Science Research in AntiDoping; 2006 Apr 13-14; Larnaca, Cyprus. WADA. 
Running head: WHY ATHLETES SAY NO TO DOPING

671 Table 1.

672

Participant Information

\begin{tabular}{|c|c|}
\hline Participants & Level of competition 673 \\
\hline Rugby $(\mathrm{n}=8 ; 8$ male $)$ & $\begin{array}{l}\text { International }(n=5 ; \text { age } \\
21-31 \text { years }) \\
\text { Premiership Club }(n=3 ; \\
\text { age } 20-26 \text { years })\end{array}$ \\
\hline Football $(\mathrm{n}=5 ; 5$ male $)$ & $\begin{array}{l}\text { International }(\mathrm{n}=5 \text {; age } \\
21-32 \text { years })\end{array}$ \\
\hline $\begin{array}{l}\text { Judo }(\mathrm{n}=8 ; 3 \text { female, } 5 \\
\text { male) }\end{array}$ & $\begin{array}{l}\text { International }(n=5 \text {; age } \\
22-29) \\
\text { Development }(n=3 \text {; age } \\
18-21 \text { years })\end{array}$ \\
\hline $\begin{array}{l}\text { Endurance sports }(n=8 ; \\
2 \text { female, } 5 \text { male })\end{array}$ & $\begin{array}{l}\text { International }(n=6 \text {; age } \\
22-29 \text { years }) \\
\text { Development }(n=2 \text {; age } \\
18-20 \text { years })\end{array}$ \\
\hline $\begin{array}{l}\text { Track and field athletics } \\
(\mathrm{n}=7 ; 2 \text { female, } 5 \\
\text { male) }\end{array}$ & $\begin{array}{l}\text { International }(\mathrm{n}=5 \text {; age } \\
21-28 \text { years }) \\
\text { Development }(\mathrm{n}=2 \text {; age } \\
18-19 \text { years }) \\
\text { Football }(\mathrm{n}=2) \\
\text { Rugby }(\mathrm{n}=1)\end{array}$ \\
\hline $\begin{array}{l}\text { Coaches }(\mathrm{n}=10 ; 10 \\
\text { male) }\end{array}$ & $\begin{array}{l}\text { Judo }(\mathrm{n}=2) \\
\text { Endurance }(\mathrm{n}=2) \\
\text { Track and field athletic } \\
(\mathrm{n}=3)\end{array}$ \\
\hline
\end{tabular}

674 
Running head: WHY ATHLETES SAY NO TO DOPING

Table 2.

Themes and sub-theme with example data extracts from interviews

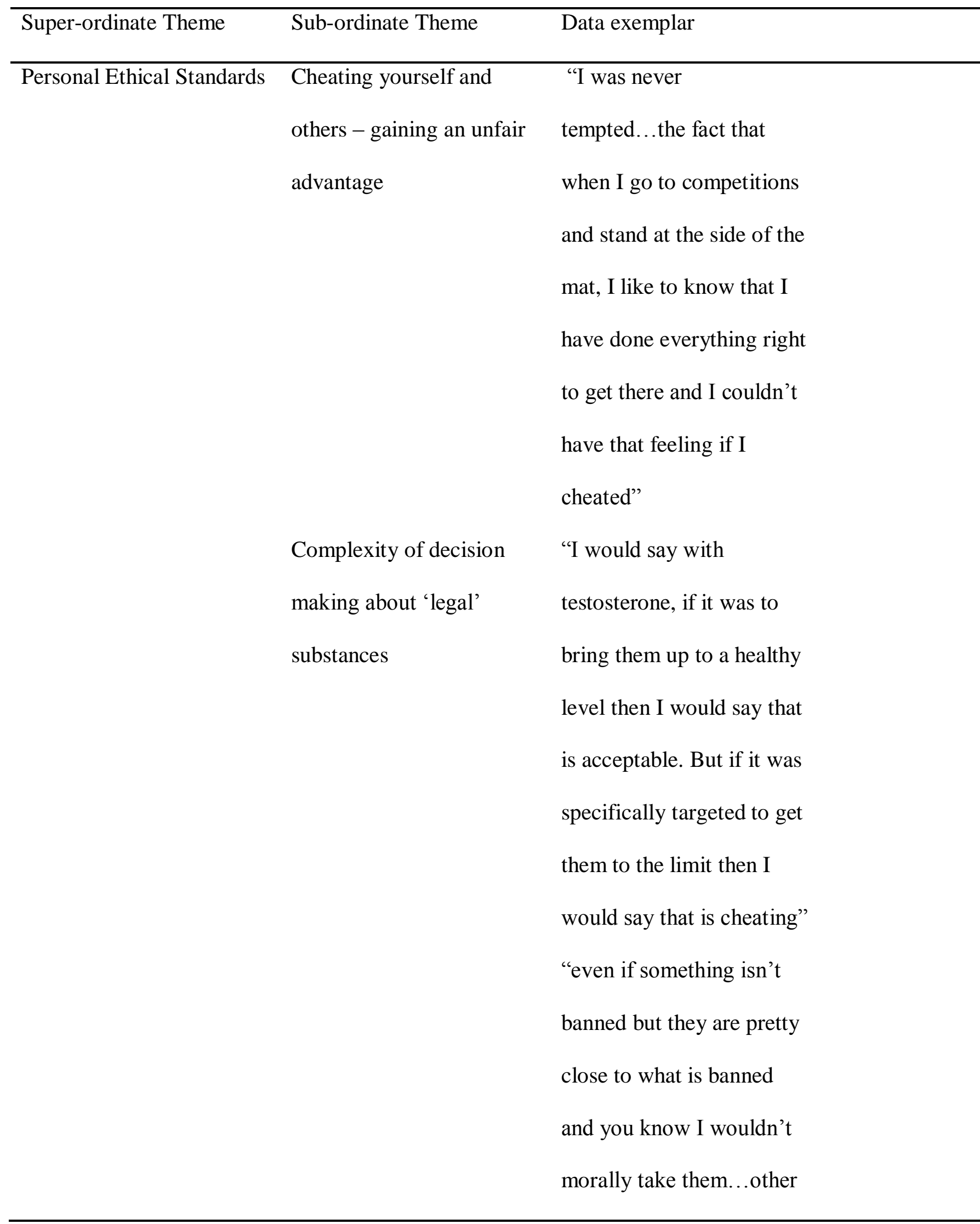


things like protein and

vitamins, they have

scientifically tested and

everyone is allowed use

them so that we all know

that is acceptable"

Personal decision guided

by moral values

Actions guided by what is

'within the rules'

Psycho-social

Environment

Letting others down
"Some things are legal and

some things aren't but I

have my own line that

goes 'that's okay and that

isn't' and that is pretty

much it"

“I don't think punching, or diving, or shirt pulling is

really cheating, it's just

part of the game and if I do

it and get caught my team

will get punished but

doping is different, that

isn't within the spirit of the

game"

"I was thinking about my

family you know, and if I

was to be caught, the

shame of it...the thought 
of my mother having to

survive that, I was a

shining star in our little

neighborhood and if I

caught you would be

letting all those people

down"

Shame and guilt

Anti-doping culture within

'their' sport / culture as a

protective mechanism

Influence of family and

parents
"I would be mortified,

embarrassed, shameful in

terms of my family, my

children"

"I don't feel like it is even

a thing in my environment,

I don't know if that is my

group, my sport or even

Great Britain but it just

isn't part of what we do"

"I think certainly my

parents are important, the

way I was brought up was

to try and if you are going

to do something do it to

the best of your ability but

to do something to the best

of your ability means to do 
it right"

Influence of Peers and "I came into judo as a

Coaches skinny 17 year old by

watching [name of judo

player] and people like

that, when they would go

off to the world

championships I was

thinking that is what I

want to do. So I learned

everything from [name of

athlete] and [name of

coach] and they would

have told me that it

[doping] is the wrong

thing to do"

Anti-doping testing and

Getting caught was not a

"I don't think that the

education

significant factor

testing is a deterrent in my

decision not to dope"

"I think that people who

dope are smart about it and

you know I'm sure the

testing procedures make

them nervous but I think a

lot of people know how to 
beat the rules"

Education not a significant "I don't think the anti-

factor

doping education stuff was

that important...by the

time I had been given the

information I had already

decided that I wasn't going

to do that sort of stuff

anyway" 
Running head: WHY ATHLETES SAY NO TO DOPING 
Running head: WHY ATHLETES SAY NO TO DOPING 